Tables show each state and region rank-ordered by its crime rates as published in the Uniform Crime Reports of the Federal Bureau of Investigation

Compiled by the Supervisor of Research Massachusetts Department of Correction

John J. Fitzpatrick

Commissioner

Publication No. 5894 approved by Alfred C. Holland, State Purchasing Agent 


\section{PLACE OF MASSACHUSETTS IN \\ NATIONAL CRIME RATE STATISTICS FOR 1970}

This report compares Massachusetts and New England with other states and regions in regard to crime rates. The rates of the pertinent crimes were obtained from the Uniform crime Reports - 1970 - issued August 31, 1971 by the Federal Bureau of Investigation.

As customary, tables in that publication list the rates per 100,000 inhabitants of every state and region for each of the following seven selected crimes:

1. Murder (including non-negligent manslaughter)

2. Forcible rape

3. Robbery

4. Aggravated assault

5. Burglary

6. Larceny of over $\$ 50.00$

7. Auto theft (including both joyriding and stealing for resale)

The figures for the quantities of these crimes are based on the number of offenses known to local police and in turn voluntarily reported by them to the F.B.I. Most, but not all, sizeable cities and towns cooperate in forwarding such statistics.

For many years it has been the F.B.I. practice to add together the rates of all of these seven selected crimes to establish an overall "Crime Index" which purports to show the amount of serious crime in an area. According to the F.B.I. the seven crimes selected "...are all serious crimes, either by their very nature or due to the volume in which they occur."*

We disagree with the notion that because an offense occurs in high volume it should be considered a serious crime. If this were so, then traffic law violations and drunkenness, which make up over $70 \%$ of the volume of Massachusetts criminal court cases,

*Uniform Crime Reports - 1970. Page 5 
should also be defined as "serious" crimes. The fact that such offenses result in a tremendous volume of social problems does not justify redefining comparatively minor crimes as serious crimes.

In fact it is because of the excessive volume of comparatively minor crimes against property included in the "Index" as compared with the limited number of more serious violent crimes against persons that the "Index" does not really indicate the true amount of serious crime. For example, the act of stealing a car for a joyride counts just as much in the "Index" as committing a murder - an obviously inappropriate equation. A comparison of the nationwide frequency of these two crimes shows that there are over fifty auto thefts for each murder. This illustrates the imbalance of the design of the "Index". Further, when we compare the total of all property crimes with all violent crimes we find that there are nearly seven times as many of the three selected property crimes as there are of the four selected violent crimes against the person. Consequently it is clear that the F.B.I. "Crime Index" is padded with altogether too many of the less serious crimes.

Overweighting the "Index" with comparatively minor crimes results in the following kinds of anomalies. Comparing Mississippi and Wisconsin we see that the former has a high murder rate of 11.5 while Wisconsin's murder rate is only 2.0. Mississippi's rate for all four violent crimes is 179.3, more than double the Wisconsin violent crime rate of 85.8 . Yet because Mississippi has a very low property crime rate this reduces Mississippi's overall Crime Index to a low 863.4 while the Crime Index for Wisconsin of 1614.4 is nearly twice as high. An index that falsely indicates that there is a much higher rate of "serious crime" in Wisconsin than in Mississippi is not very accurate. Or consider another example. Should a state have a $100 \%$ increase in murders this could be counterbalanced by a 1\% drop in burglaries and the overall "Crime Index" would then show a decrease in the amount of serious crime.

Because the F.B.I. "Crime Index" does not give a true picture of the amount of serious crime we omit the overall "Index" figures from our table and do not use them in our comparisons. However the Uniform Crime Reports do show additional sub-total figures, on the one hand aggregating the rates for the four violent crimes against the persons while 
on the other hand separately aggregating the rates for the three property crimes. This breakdown into two separate sub-groups avoids adding a rate for a violent crime like murder, which usually occurs less than eight times per hundred thousand, together with a rate for property crime like burglary, which may occur over a thousand times per hundred thousand people. In other words having two separate sub-totals permits grouping together similar offenses which have less markedly disparate rates. However, even within these more similar groups there are still variations of frequency. For example, within the Violent crime sub-group, robbery occurs twenty times as often as murder. However because the distortions of the overall "Crime Index" are considerably minimized the sub-totals of Violent Crimes and Property Crimes are utilized in this paper.

To obtain a better comparison of Massachusetts with all other states we rank ordered all state rates for each crime. The state with the lowest rate was placed number one at the top of the list for that crime, the next lowest was located number two and so on. This of course resulted in the state with the highest rate for a crime being ranked fiftieth at the bottom of the column for that crime. Table I appended to this paper shows the resulting rank order of each state for each offense, as well as the rank of each state with regard to the total for all Violent crimes as well as the total for all property crimes. The rank order of nine regions of the United States (see Footnote 1) are also shown.

Footnote 1

$\underline{\text { Region }}$

1. New England

2. Middle Atlantic

3. East North

Central

4. West North

Central

5. South Atlantic

6. East South

Central

7. West South

Central

8. Mountain

9. Pacific
States Included

Conn, Maine, Mass., N.H., R.I., Vermont

New Jersey, New York, Pennsylvania

Ill., Ind., Mich., Ohio, Wisc.

Iowa, Kans., Minn., Mo., Neb., No. \& So. Dakota Del., Fla., Ga., Md., N. \& S. Car., Va., W. Va.

Alabama, Kentucky, Mississippi, Tennessee

Arkansàs, Louisiana, Oklahoma, Texas

Ariz., Colo., Idaho, Mont., Nev., N. Mex., Utah Wyo.

Alaska, Calif., Hawaii, Oregon, Washington 
To supplement Table I which shows the crime rates and rank order of all states and regions for the one year of 1970 we prepared an additional table based on information taken from reports of previous years. This new Table II shows the crime rates over a five year period for the United States, for New England and for Massachusetts - but not for every state or other regions. This table indicates that crime rates have increased every year and also shows the rank order changes, if any, from year to year.

Inspection of the regional rankings in Table I shows that in regard to the total of the four violent crimes against persons New England has the lowest rate and consequently is ranked number 1 above the eight other areas of the country. Looking specifically at each individual offense we find that New England has the number 1 rating for the crimes of murder, rape and aggravated assault and the number 2 rating for robbery.

However, with regard to crimes against property New England's rate for the aggregate total of these three crimes drops to 6 th. As to each individual offense New England ranks 5th in burglary, $3 r d$ in larceny and 9th, or last, for auto theft. (It is the bad ratings of Massachusetts and Rhode Island that drop the New England region into the bottom rank for auto theft.)

Comparison of the state of Massachusetts with all other states in regard to violent crimes shows that it has a somewhat better rank (20th) than the median for all states when the rates of all four of the violent crimes are aggregated. As to each individual crime Massachusetts ranks 13th in murder, 18th for forcible rape, 32nd for robbery and 16th for aggravated assault.

In regard to crimes against property our state does not compare as well. It ranks poorer (4lst) than the median for the aggregate of the three property crimes as well as worse than the median for each individual property offense as follows: burglary (37th) larceny (27th) and auto theft (50th).

The median for fifty states would of course be located between the 25 th and 26 th ranks. A national average, being a different measure than the median, provides another standard. When we compare our state crime rates with the national averages for each crime the result shows Massachusetts in a sljghtly better light. Although Massachusetts has a poorer rate than the medians 
for four (robbery, burglary, larceny and auto theft) of the seven crimes, when we compare with national averages there are only two offenses (burglary and auto theft) for which the Massachusetts rate is worse. In other words, Massachusetts has a lower crime rate than the national average for three of the four violent crimes but has a lower rate than average for only one (larceny) of the three property crimes.

Turning to Table II which shows trends over a five year period we see that, in regard to violent offenses, New England has consistently been the best region. However, with regard to crimes against property we find that New England regularly has poorer ratings. Year after year the more urban states of connecticut, Rhode Island and Massachusetts drag New England down in the property crime rate rankings while the more rural states of Maine, New Hampshire and Vermont are usually among the top ten most crime free states in the country for property crimes as well as for violent crimes against persons.

Looking at the section in Table II which shows the Massachusetts figures we see that for crimes against the person Massachusetts is usually ranked well above the median except for robbery. However, for crimes against property it is consistently ranked below the median. The Massachusetts rankings for the offenses of murder, robbery, aggravated assault and auto theft have hovered near the same rank each year. However, in regard to the offenses of rape, burglary and larceny we see that Massachusetts has dropped several places since 1966. Although we noted that the Massachusetts ranking for auto theft has remained unchanged it should be emphasized that as our rank is consistently 50th it would be impossible to drop to any lower rank. Whatever officials in Massachusetts have been doing, if anything, to reduce the incidence of reported auto theft certainly has not been very successful up through 1970! 
1970 Rates per 100,000 Inhabitants

\begin{tabular}{|c|c|c|c|c|c|c|c|c|c|c|c|c|c|c|c|c|c|c|c|}
\hline & & & & & 6 & $=T E D$ & $C R_{1}$ & IMES & vs & $P E$ & $O N$ & 15 & a & $P 0$ & $B T$ & $r Y$ & & & \\
\hline $\begin{array}{l}\text { Ranh } \\
\text { Order }\end{array}$ & & $\begin{array}{r}\text { CRIMEE } \\
\text { PERS }\end{array}$ & $\begin{array}{l}\text { ES Vo } \\
\text { SONS }\end{array}$ & $\begin{array}{l}\text { Murder } \\
\text { non nesl.n }\end{array}$ & & $\begin{array}{l}\text { For } \\
\text { Ra }\end{array}$ & & Rob b & bepy & $\begin{array}{l}\text { Aggr } \\
\text { As: }\end{array}$ & $\begin{array}{l}\text { ted } \\
\text { it }\end{array}$ & $\begin{array}{l}\text { CRIM } \\
\text { PROP }\end{array}$ & $\begin{array}{l}\text { ES V V } \\
\text { EATY }\end{array}$ & Burgl & lary & $\begin{array}{l}\text { Larce } \\
\text { c5o and }\end{array}$ & $\begin{array}{l}\text { eny } \\
\text { dover) }\end{array}$ & & \\
\hline & & Area & Rate & Area & Rate & Area & Rate & Area & Rake & Area & Rate & Area & Rote & Area & Rate & Area & Rate & Area & Rate \\
\hline & & U.S. & 360.0 & U.S. & 7.8 & U.S. & 18.3 & u.s. & 171.5 & U.S. & 162.4 & U.s. & 2380.5 & U.s. & 1067.7 & I.S. U.S. & 858.4 & Us. & 453.5 \\
\hline & 1 & New Eng. & 170.7 & N $k_{k} E_{n g}$. & 3.1 & Mow $E_{n g}$ & $9.7 t$ & E.S. Cont & 60.21 & New Eng & 03.6 & ESo. Cent. & 1463.1 & ES So Cent & 690.3 & E. So. Cant & 523.4 & E. S. $\{(\mathrm{an})$. & 249.4 \\
\hline$R$ & 2 & W.NoCent. & $2 / 5.2$ & W.M. Lent & 4.9 & Mis. Allow' & 13.7 & New Eng. & 74.2 & WNoccent & 95.8 & Who cont. & 1828.0 & Wo. Cent & 797.9 & & 696.0 & & 334.1 \\
\hline$E$ & 3 & E. S. Cent'. & t. 250.7 & Anarific & 6.3 & W. Ha Cont & 14.7 & Movatain & 90.6 & E. Ho. Cent & 1396 & W. Sa Cent. & t. $2103.6 \|$ & E. Wo Cant. & 936.6 & New Eng & 727.45 & So Atlent. & 346.2 \\
\hline G & 4 & Mountain & 275.1 & Mountain & 6.7 & E. So Cent & 14.7 & Wrelo. Cont & 99.7 & Mid. AtI. & 150.0 & E. No [ent. & $.2175 .9 \|$ & W. Sa Cant? & 1008.2 & W. 6 . Cont. & 747.0 & K. $\int_{0}(\mathrm{an})$. & 3484 \\
\hline 1 & 5 & W.So Cent. & 1. 335.7 & Mid. Allant & 6.7 & E.No Cent & 18.0 & W.So.Cand & $1 / 6.0$ & Mountain & 1555 & 50. Atlaat? & $2236.6 \| A$ & New Eng & 1013.6 & & 785.5 & Mountain & +29.1 \\
\hline 0 & 6 & $E N_{0} \cdot C_{a_{n}} t$. & 367.0 & E.Mo.Cent. & 7.2 & So. Attlentic & 18.0 & Santlo & 168.5 & E.So.Cent & 165.1 & Naw Eng. & $2383.1 \| 5$ & So. Atlant. & $10+3.6$ & So Atlant? & 846.8 & E.N. So. Sent. & 153.8 \\
\hline N & 7 & Pacific & $4 / 2.6$ & E So Cent. & 10.6 & w.s. & 20.3 & Pacific & 178.4 & W. So. Cont & 188.8 & Mid Atie. & 2485.3 & Mid Allad. & 1082.0 & Mid Allan & 853.8 & Mid Atlont & 549.5 \\
\hline 5 & 8 & So. Allaat? & 475.4 & W.So. Cent. & 10.7 & & 23.0 & & 202.3 & Pacific & 197.2 & Mountain & $=2688.7 \|$ & Mountain & 1176.1 & Mountain & 10855 & Pacific & 614.3 \\
\hline & 9 & Mid. All. & 453.8 & So.Atlantk & 11.8 & Pacific & 30.8 & Mir. Atileal. & 283.4 & So. A+1. & 227.0 & Pacific & 3597.6 & Pacific & 1656.0 & Pacific & 1327.4 & New Eng & 642.1 \\
\hline & 1 & No Dak. & 34.2 & No Dak. & 0.5 & R.I. & 3.6 & No.Dak & $6.5 \mid \lambda$ & No. Dak & 21.0 & Miss & $68 \%, 1$ & No Dak. & $286 \%$ & Miss. & $25 * 6$ & Miss & 78.4 \\
\hline 5 & 2 & N Hamp. & 56.0 & Vermont & 13 & W Hamp & $6.0 \mathrm{r}$ & Kermont & 7.6 & N. Hamp & 35.9 & No. Dak. & 846.1 & Miss & 351,2 & Vermonl & 340.7 & & 91.0 \\
\hline$T$ & 3 & Vermont & 74.0 & Maine & 15 & No. Dak & 6.2 & N Hamp. & 12.1 & Iowa & 42.8 & $w v_{a}$. & 835.0 & W. $V_{a}$ & 388.5 & Maine & 350.3 & w. & 92.4 \\
\hline A & 4 & Iowa & 79.3 & Iowa & 1.9 & Lowa & 6.2 & Maine & 126 & Hawaii & 43.1 & Moine & 1058.0 & So DaK & 471.3 & W. Va & $35 * 0$ & So. Dak & 93.7 \\
\hline$T$ & 5 & Maine & 82.8 & N. Hamp. & 2.0 & Wisc. & 6.7. & So. Dak & 17.1 & Wisce. & 440 & S. Dah & 1059.7 & Neb. & 504.5 & SPenn. & 3947 & Ark & 1090 \\
\hline E & 6 & Wise. & 85.8 & Wisc. & 2.0 & W Va. & 6.7 & Miss. & 19.0 & & 51.2 & N Hamp. & 1136.7 & Iowa & 507.3 & $N$ Hamp & 399.1 & Vermont & 1239 \\
\hline & 7 & So Dak & 92.5 & Minn & 2.0 & Maine & 7.0 & Idaho & 20.5 & Vermont & 546 & Vermont & 1195.1 & Wisc. & 538.5 & 5 No. Dak & 434.6 & Idaho & 144.2 \\
\hline & 8 & Mont. & 1115 & $\mathrm{Neb}$ & 3.0 & Misg. & 8.91 & Wyo. & 22.0 & So. Dak. & 60.5 & Penn & 1329.1 & Mains & 562.4 & So.Dak & 494.7 & Maine & 46.1 \\
\hline & 9 & Wyo. & 113.1 & Mont & 3.2 & Conn & $9.1 \%$ & Mont & 22.3 & Main & 61.7 & Neb. & 1333.1 & N.Hamp. & 565.6 & stenn. & 517.4 & 4 No.Cat. & 150.6 \\
\hline & 10 & Hawori & 121.8 & R.I. & 3.2 & Nob. & 9.3 & $W . V_{a}$ & 27.3 & Uiah & 70.3 & Iowa & 1356.0 & Mont. & 593.7 & 7 Neb & & Wyo & 154.6 \\
\hline & 11 & Idatio & 123.3 & Utah & 3.4 & Minh & 9.7 & Iowa & 28.5 & Swo. & 73.1 & $A+k$. & 1381.4 & Penn & 594.3 & 3 AI & 583.2 & & 172.0 \\
\hline & 12 & W. Va & 123.7 & Cann. & 3.5 & f Vernoat & 10.3 & Wisc. & 33.1. & Mont. & 75.5 & Wisce & 1428.6 & & 645.9 & & 587.4 & & 175.0 \\
\hline & 13 & Utah & 137.7 & MASS & 3.5 & 5 Mont & 10.5 & $A+k$ & 45.6 & w. Va & 83.5 & No. Cart & 1498.9 & Idato & 673.6 & I & 596.0 & Wise & 217.6 \\
\hline & 14 & Minh & 152.0 & $\overline{\text { Wash }}$ & 3.5 & Utah & 10.9 & No.Car. & 49.2 & Idaho & 85.8 & Mont. & 1525.3 & Ark & 685.1 & 5.6 & 6293 & Mont. & 21.8 \\
\hline & 15 & Conn. & 170.4 & Hawroii & 3.6 & So. Dak & 11.1 & Ala. & 50.3 & Conn. & 87.4 & Rla. & 1569.7 & Kenty. & 703.9 & No.Cat. & 639.7 & $A \mid a$ & 2235 \\
\hline & 16 & Miss. & 179.3 & So Dak. & 3.8 & Pann. & 11.3 & Utah & 53.1 & MASS & 87.9 & Tenn. & 1613.4 & No Cer. & 708.6 & 6 Kenty & 651.9 & So.Car. & 46.5 \\
\hline & 17 & Neb. & 184.1 & Idaho & 4.6 & Hawoii & 11.8 & $O k / a$ & 53.8 & Penn & 89.6 & Wyo. & 1632.0 & Vermont & 730.6 & Wisc. & 672.5 & Kans. & 56.6 \\
\hline & 18 & $0 \mathrm{k} / \mathrm{a}$. & 197.8 & Ore. & 46 & 6 MASS & 12.0 & Neb. & 57.3 & Ind. & 95.3 & Idaho & 1661.8 & Ala & 763.1 & Ior & 673.7 & okla. & 75.4 \\
\hline & 19 & Kans. & 202.8 & Kans. & 4.8 & Wyo. & 12.3 & So. Cor. & 60.0 & N. Jers. & 99.0 & Kent. & 1702.2 & III. & 765.4 & Mo. & 684.2 & Tenn & 289.3 \\
\hline & 20 & MASS & 202.9 & Ind. & 8 & Id daho & 12.3 & Hawaii & 63.3 & Wash. & 106.3 & okla. & 1753.2 & Alaska & 789.9 & Okla. & 887.4 & 4 Neb. & 291.9 \\
\hline & 21 & R. I. & 204.7 & Penn & 5.3 & No.Cor. & 12.6 & $N \cdot M$ & 66.1 & Kans. & 108.5 & So.Car. & 1781.6 & & 793.3 & & & & \\
\hline & 22 & Penn. & 212.2 & Wyo. & 5.7 & N. Jers. & 12.9 & Cor & 70.4 & Neb & 114.5 & III. & 1879.2 & $M_{1}$ & 801.7 & 7 Mont. & 09.8 & Ga & 08.6 \\
\hline & 23 & Wash & 221.3 & N. Jers & 5.7 & Kent & 13.7 & Aloske & 71.8 & Ohio & 115.9 & va. & 1890.2 & $v a$. & 805.6 & $6 L$ & 716.4 & Utah & 16.3 \\
\hline & 24 & $A+k$. & 222.3 & Okle. & 5.9 & Kans & 14.5 & $5 \in \operatorname{Kent}$ & 72.8 & R.I & 119.6 & $G a$. & 1902.2 & Tenn & 806.7 & 7 ohio & 745.8 & ore. & 32.8 \\
\hline & 25 & Kent & 222.3 & W. Va & 6.2 & $V a$ & 15.4 & Kans. & 75.1 & $0 \mathrm{k} / \mathrm{a}$ & 122.4 & Kans. & 1941.1 & Ohio & 853.9 & Ind. & 756.1 & Penn. & 340.1 \\
\hline & 26 & Ind. & 225.5 & Colo & 6.2 & Tenn & 15.5 & $R . I$ & 78.3 & & 124.7 & Minn. & 1951.4 & Ind. & 8600 & & 87.2 & $M i$ & 5.7 \\
\hline & 27 & Del. & 256.0 & hio. & 6.6 & 6 N.york & 15.5 & Tenn. & 82.0 & $D=1$. & 130.3 & La. & 1991.2 & Kans. & 881.7 & MASS & 788.9 & Kenty & 46.3 \\
\hline & 28 & ore. & 256.9 & Del. & 6.9 & okla. & 15.6 & Minn. & 89.1 & Ore. & 130.7 & Ind. & 2045.0 & La. & 890.0 & Tex & 798.7 & 7 Wash. & 361.8 \\
\hline & 29 & $v a$ & 259.0 & Colif & 6.9 & Ohio & 160 & Va. & 92.0 & Miss & 139.8 & Ohio & 2092.4 & $G a$. & 891.9 & & 802.7 & La. & \\
\hline & 30 & Tenn. & 274.9 & N. York & 79 & $G a$. & 16.1 & Wash. & 93.5 & $V a$ & 143.2 & Utah & 2235.0 & So. Car. & 905.8 & Minn & 804.0 & o N.Mex & 3922 \\
\hline & 31 & Alaska & 278.0 & & 8.4 & 4 Del. & 16.8 & & & & 149.5 & Texas & 2344.3 & Utah & $9 / 5.0$ & Wyo. & 821.6 & & \\
\hline & 32 & Ohio & 284.3 & Tenn. & 8.8 & $A+k$ & 17.1 & MASS & 99.5 & Mo. & 167.0 & Mo. & 2359.1 & Del. & 979.0 & Conn. & 836.1 & Fla. & 396.6 \\
\hline & 33 & So. Car. & 285.2 & Ner. & 8.8 & So. Cat. & 17.1 & $D \in I$. & 102.0 & Alaske & 167.8 & Conn. & 2404.5 & R.I. & $10 / 89$ & R.I. & 43.2 & Ind. & 428.9 \\
\hline & 34 & N. Jers. & . 287.1 & Mich & 8.9 & Ind & 17.9 & ore. & 102.5 & Tenn. & 168.6 & Alaska & 2412.5 & N. Jero & 1041.4 & $+I_{d}$ & 34.0 & $M_{i}$ & 459.0 \\
\hline & 35 & N.Mex. & 292.8 & Md & 9.2 & Wash & 18.0 & Penn. & 106.0 & $G a$ & 177.3 & N.Jers. & 2457.1 & Md & 1051.2 & & 858.2 & Conn & 482.2 \\
\hline & 36 & Ala. & 295.7 & N.Mex & 9.4 & ore. & 18.0 & & 107.5 & & 181.7 & Del. & 2460.1 & & $108 \% .2$ & & & & \\
\hline & 37 & $G a$. & 304.5 & Ariz & 9.5 & 5 Ala. & 18.5 & Ariz. & 120.2 & Mich. & 182.6 & N. Mex. & 2572.6 & MASS & 1134.1 & & 1003.8 & & \\
\hline & 38 & Colo. & 356.7 & & 9.6 & Ner. & 19.6 & Colo. & 129.1 & Colo. & 1854 & R.I. & 2721.1 & & & N.Max. & 1039.1 & & \\
\hline & 39 & Texas & 361.5 & $A+k$. & 10.1 & III. & 20.4 & Texas & 134.1 & $I I I$. & 1868 & Md. & 2722.1 & N.Mex. & 1141.5 & Ala & 1071.2 & Mo. & 53 \\
\hline & 40 & No.Car. & & & 10.7 & Texas & 21.0 & La. & 140.8 & Sacar. & 193.4 & ore. & 2730.4 & Texas & 1151.3 & Ariz & 1080.7 & Del. & 544.6 \\
\hline & 41 & Ariz. & 370.3 & & 11.1 & N.Mex. & 21.7 & Ohio & 145.9 & Texas & 194.8 & MASS & 2801.1 & & 1273.4 & & 1123.3 & & 547.6 \\
\hline & 42 & Ner. & 398.6 & No. Car. & 11.1 & F/a. & 22.2 & N.Jers. & 169.4 & N. Mex. & 195.7 & Wash & 2935.2 & colo. & 1380.9 & Ore. & 1124.1 & Haska & 551.3 \\
\hline & 43 & Mo. & 405.9 & Miss & 11.5 & 5 Mich & 22.9 & F/a. & 186.1 & N. Yook & 209.3 & Ariz & 3074.9 & N. York & 1414.2 & Wash. & 1129.0 & N Jers. & 557.4 \\
\hline & 44 & $L a$. & 413.5 & Texas & 11.6 & 6 La. & 23.1 & Nev. & 188.4 & Ariz. & 213.7 & Mich. & 3096.3 & Wash. & 1444.5 & Fla & 1143.1 & Hewaii & 581.1 \\
\hline & 45 & |III. & 467.9 & Ala. & 11.7 & Md & 23.9 & Mo. & 200.8 & $A / a$. & 215.2 & Fla. & 3101.5 & Hawaii & 1456.1 & Mich & 1143.8 & Colo. & \\
\hline & 46 & Calif. & 474.8 & & 11.7 & & 26.1 & & & Cal & 225.9 & & 3246.0 & Ariz. & 1493.0 & N.York & 1149.6 & ser. & 660.7 \\
\hline & 47 & F/a. & 498.2 & Alaska & 12.2 & 2) Ariz. & 27.0 & III. & & La & 2378 & Hawaii & 3274.4 & Mich & & Sl Hawaii & 1237.2 & N. York & 682.2 \\
\hline & 48 & Mich. & 562.0 & $F / a$. & 12.7 & Mo. & 27.4 & Md. & & $M d$ & 253.3 & Colo. & 3305.5 & Fla. & 1561.8 & Ner. & 1276.3 & Calif. & 689.2 \\
\hline & 49 & Md. & 624.9 & So. Car. & 14.6 & 6) $\mathrm{Ca}$ & 35.1 & Mich. & 348.4 & Fla. & 277.2 & Ner. & 3597.6 & Ner. & 1660.6 & Colo. & 1336.1 & R.I. & 859.0 \\
\hline & so & & & & & & 36.0 & N.York & 443.3 & No.Car. & 289.6 & Calif. & 3832.1 & Calif. & 1753.0 & Calif & 1389.9 & Mass & 878.1 \\
\hline
\end{tabular}


TABLE II CRIME RATES of the UNITED STATES, NEW ENGLAND and MASSACHUSETTS from 1966 through 1970 (Rates are per 100,000 Inhabitants)

\begin{tabular}{|c|c|c|c|c|c|c|c|c|c|c|c|c|c|c|c|c|c|c|}
\hline \multirow{2}{*}{$\begin{array}{l}\text { AREA } \\
\text { and } \\
Y E A R\end{array}$} & \multicolumn{2}{|c|}{$\begin{array}{l}\text { TORAL CAINES } \\
\text { US PERSONS }\end{array}$} & \multicolumn{2}{|c|}{ MURDER } & \multicolumn{2}{|c|}{ RAPE } & \multicolumn{2}{|c|}{ ROSAERY } & \multicolumn{2}{|c|}{\begin{tabular}{|c|} 
AGGAAVATED \\
ASSAULT
\end{tabular}} & \multicolumn{2}{|c|}{$\begin{array}{l}\text { TOTAL CRIMES } \\
\text { YS PROTERTY }\end{array}$} & \multicolumn{2}{|c|}{$B U R G L A R Y$} & \multicolumn{2}{|c|}{$\angle A R C E N Y$} & \multicolumn{2}{|c|}{ Auto rhart } \\
\hline & & Rate & & Rete & & Rale & & Rate & & Rate & & Rate & & Pale & & Rate & & Rate \\
\hline $\begin{array}{c}\text { UNITED STATES } \\
1966\end{array}$ & & 215.2 & & 5.6 & & 12.9 & & 78.3 & & 118.4 & & 1440.8 & & 699.6 & & 456.8 & & 284.4 \\
\hline U.S. 1967 & & 249.9 & & 6.1 & & 13.7 & & 102.1 & & 128.0 & & 1671.7 & & 811.5 & & 529.2 & & 331.0 \\
\hline U.S. 1968 & & 294.6 & & 6.8 & & 15.5 & & 131.0 & & 141.3 & & 1940.2 & & 915.1 & & 636.0 & & 389.1 \\
\hline U.S. 1969 & & 324.4 & & 7.2 & & 18.1 & & 147.4 & & 151.8 & & 2146.7 & & 965.6 & & 749.3 & & 431.8 \\
\hline \multirow[t]{2}{*}{ US. 1970} & & 360.0 & & 7.8 & & 18.3 & & 171.5 & & 162.4 & & 2380.5 & & 1067.7 & & 859.4 & & 453.5 \\
\hline & $R_{e, n k}$ & & $R_{e n}$ & & Ranak & & $\begin{array}{c}\text { Pank } \\
1\end{array}$ & & $\frac{P \operatorname{mon}}{1}$ & & Penk & & $\begin{array}{c}R e n k \\
z\end{array}$ & & $\begin{array}{c}\operatorname{acm}{ }^{2} \\
2\end{array}$ & & $\sin k$ & \\
\hline $\begin{array}{c}\text { NEW ENGLAND } \\
1966\end{array}$ & 1 & 88.9 & 1 & 2.1 & 1 & 5.9 & 1 & 30.7 & 1 & 50.1 & * & 1301.7 & 3 & 576.2 & 3 & 336.2 & 8 & 389.3 \\
\hline N.E. 1967 & 1 & 103.8 & 1 & 2.4 & 1 & 6.3 & 1 & 37.0 & 1 & 58.1 & $\star$ & 1507.1 & 3 & 663.4 & 2 & 373.2 & 9 & 471.5 \\
\hline N.E. 1968 & 1 & 131.6 & 1 & 2.9 & 1 & 7.9 & 2 & 52.6 & 1 & 68.2 & 6 & 1909.5 & 5 & 831.0 & 3 & $5 / 2.6$ & 9 & 565.9 \\
\hline NE. 1969 & 1 & 152.2 & 1 & 3.0 & 1 & 8.8 & 2 & 65.6 & 1 & 74.8 & 6 & 2166.9 & 6 & 920.5 & 4 & 637.0 & 9 & 609.4 \\
\hline \multirow[t]{2}{*}{ N.E. 1970} & 1 & 170.7 & I & 3.1 & 1 & 9.7 & 2 & $7 \nvdash 2$ & 1 & 83.6 & 6 & 2383.1 & 5 & 1013.6 & 3 & 727.4 & 9 & 642.1 \\
\hline & $\begin{array}{c}\text { Renk } \\
2\end{array}$ & & Penth & & $\begin{aligned} R \operatorname{lanh} \\
2\end{aligned}$ & & 2 & & $\operatorname{Rank}$ & & $\begin{array}{c}\operatorname{Ranh} \\
2\end{array}$ & & 2 & & $\begin{array}{c}2 \operatorname{sank} \\
2\end{array}$ & & $\begin{array}{c}R_{a}, k \\
2\end{array}$ & \\
\hline $\begin{array}{c}\text { MASSACH USETIS } \\
1966\end{array}$ & $\star$ & 115.3 & 13 & 2.4 & 10 & 6.4 & 32 & 46.0 & 15 & 60.5 & $\star$ & 1539.0 & 32 & 619.0 & 24 & 368.2 & 50 & 551.8 \\
\hline MASS 1967 & $\star$ & 127.6 & 13 & 2.8 & 14 & 7.6 & 29 & 52.0 & 17 & 65.2 & * & 1735.2 & 28 & 675.5 & 20 & 3923 & 50 & 667.4 \\
\hline MASS. 1968 & 20 & 164.0 & 16 & 3.5 & 16 & 9.5 & 30 & 74.3 & 18 & 76.7 & 41 & 2220.6 & 33 & 868.3 & 29 & 545.7 & 50 & 806.6 \\
\hline MASS. 1969 & 23 & 187.9 & 16 & 3.5 & 13 & 10.8 & 33 & 90.6 & 17 & 82.9 & 38 & 2552.3 & 36 & 1032.6 & 27 & 661.0 & 50 & 858.8 \\
\hline MASS. 1970 & 20 & 202.9 & 13 & 3.5 & 18 & 12.0 & 32 & 99.5 & 16 & 87.9 & 41 & 2801.1 & 37 & 1134.1 & 27 & 788.9 & 50 & 878.1 \\
\hline
\end{tabular}

SOUACE Uniform Crime Reports 1966 through 1970

N.N. Cochrane

FOOTNOTES 1 INDICATES RANK WITHIN 9 REGIONS

2 INDICATES RANK WITHIN 50 STATES * INOICATES RANK ORDER NOT COMPUTED 
